On the Barzilai and Borwein Choice of Steplength for the Gradient Method

Marcos Raydan

May 1990

(revised October, 1991)

TR90-11 



\title{
On the Barzilai and Borwein Choice of Steplength for the Gradient Method*
}

\author{
Marcos Raydan ${ }^{\dagger}$
}

October 1991

\begin{abstract}
In a recent paper, Barzilai and Borwein presented a new choice of steplength for the gradient method. Their choice does not guarantee descent in the objective function and greatly speeds up the convergence of the method. We derive a relationship between the gradient method for minimizing a quadratic function and the shifted power method. This relationship allows us to establish the convergence of the Barzilai and Borwein method when applied to the problem of minimizing any strictly convex quadratic function (Barzilai and Borwein considered only 2-dimensional problems). Our point of view also allows us to explain the improvement obtained by using this new choice of steplength.
\end{abstract}

${ }^{*}$ During the course of this research the author was a graduate student in the Department of Mathematical Science at Rice University, Houston, Texas. He gratefully acknowledges Universidad Central de Venezuela for support during this period of time.

tDepartment of Mathematics, University of Kentucky, Lexington, Kentucky, 40506 



\section{Introduction}

In order to solve the unconstrained minimization problem, we consider the nonlinear equations problem :

$$
\text { find } x_{\star} \in I^{n} \text { such that } \nabla f\left(x_{\star}\right)=0 \text {, }
$$

where $f: I R^{n} \rightarrow I R$. The numerical solution of (1) is usually iterative, moving at each iteration from an estimate $x_{c}$ of $x_{\star}$ to a better estimate $x_{+}$. In many algorithms, each iteration involves the calculation of a quasiNewton step, $s_{Q N}=-A_{c}^{-1} \nabla f\left(x_{c}\right)$, where $A_{c} \epsilon I R^{n \times n}$ is an approximation of the Hessian of $f$ at $x_{c}$. After each iteration the current $A_{c}$ is updated to $A_{+}$, an approximation of the Hessian of $f$ at $x_{+}$. The approximation usually is chosen to satisfy the secant equation,

$$
A_{+} s_{c}=y_{c}
$$

where $s_{c}=x_{+}-x_{c}$ and $y_{c}=\nabla f\left(x_{+}\right)-\nabla f\left(x_{c}\right)$.

In the one dimensional case the secant equation completely determines $A_{+}$; however if $n>1$, then many matrices will satisfy the secant equation. So, in addition to obeying (2), the update $A_{+}$must be further restricted to a set of matrices that have desirable properties, See Denuis and Schnabel [3].

Barzilai and Borwein in [2] considered a related but somewhat different approach. They observed that the scalar $\alpha_{+} \epsilon I R$ that uniquely solves the overdetermined linear system $y_{c}=\alpha_{+} s_{c}$ in the least squares sense is given by

$$
\alpha_{+}=\frac{s_{c}^{t} y_{c}}{s_{c}^{t} s_{c}}
$$

if $s_{c} \neq 0$. Hence by restricting the update matrix in the quasi-Newton method to the class of scalar multiples of the identity and then asking that the secant equation be satisfied in the least squares sense they devised the following algorithm

\section{Algorithm 1 (Barzilai and Borwein Method)}

Given $x_{0} \in I R^{n}, \alpha_{0} \in I R$

For $k=0,1, \ldots,($ until convergence $) d o$

$$
\text { 1. Set } s_{k}=-\frac{1}{\alpha_{k}} \nabla f\left(x_{k}\right)
$$


D. Set $x_{k+1}=x_{k}+s_{k}$

3. Sel $y_{k}=\nabla f\left(x_{k+1}\right)-\nabla f\left(x_{k}\right)$

4. Set $\alpha_{k+1}=\frac{s_{k}^{t} y_{k}}{s_{k}^{t} s_{k}}$

End do

This algorithm requires only $\mathrm{O}(n)$ floating point operations per iteration in arddition to the gradient evaluation.

If we consider problem (1) when $f(x)=\frac{1}{2} x^{t} A x-b^{t} x+c$ is a quadratic function and $\mathrm{A}$ is a symmetric positive definite (SPD) matrix, then $\alpha_{+}$in (3) becomes

$$
\alpha_{+}=\frac{s_{c}^{t} A s_{c}}{s_{c}^{t} s_{c}}
$$

and Algorithm 1 becomes

\section{Algorithm 2 (Barzilai and Borwein Method for Quadratics)}

Given $x_{0} \subset \mathbb{R}^{n}, \alpha_{0} \in \mathbb{Z}$

For $k=0,1, \ldots$, (until convergence) do

1. Set $s_{k}=-\frac{1}{\alpha_{k}} \nabla f\left(x_{k}\right)$

2. Set $x_{k+1}=x_{k}+s_{k}$

3. Set $\alpha_{k+1}=\frac{s_{k}^{t} A s_{k}}{s_{k}^{t} s_{k}}$

End do

In the quadratic case, $\alpha_{k+1}$ turns out to be the Rayleigh quotient of $\mathrm{A}$ at the vector $s_{k}$. Since $\mathrm{A}$ is $\mathrm{SPD}$,

$$
0<\lambda_{\min } \leq \alpha_{k} \leq \lambda_{\max } \quad \text { for all } k,
$$

where $\lambda_{\min }$ and $\lambda_{\max }$ are respectively the smallest and largest eigenvalues of A. And so, in step 1 there is no danger of dividing by zero.

Barzilai and Borwein [2] also observed, by symmetry, that the scalar $\hat{\alpha}_{+}$ that uniquely solves the overdetermined linear system $\frac{1}{\hat{\alpha}_{+}} y_{c}=s_{c}$ in the least squares sense is given by

$$
\hat{\alpha}_{+}=\frac{y_{c}^{t} y_{c}}{s_{c}^{t} y_{c}}
$$


if $s_{c}^{t} y_{c} \neq 0$. In the quadratic case, $\hat{\alpha}_{+}$becomes

$$
\hat{\alpha_{+}}=\frac{s_{c}^{t} A^{2} s_{c}}{s_{c}^{t} A s_{c}},
$$

which is the Rayleigh quotient of $A$ at the vector $\sqrt{A} s_{c}$. Hence, $\hat{\alpha}_{+}$also satisfies (5).

In the rest of this paper, we will only consider the Barzilai and Borwein method with the choice of $\alpha_{+}$defined by (3) in the general case and by (4) in the quadratic case. The reason for this is that all results established for Algorithm 2 with the choice $\alpha_{+}$also hold with the choice $\hat{\alpha}_{+}$since the method has the same properties in either case.

Notice that, in the Barzilai and Borwein gradient method, the search direction is always the negative gradient of $f$ at $x_{c}$ as in the gradient method, but the choice of steplength is not the standard choice. In fact, Algorithm 2 would be the steepest descent method for quadratics if we changed (4) to

$$
\alpha_{+}=\frac{g_{+}^{t} A g_{+}}{g_{+}^{t} g_{+}},
$$

where $g_{+}=\nabla f\left(x_{+}\right)$. Since the vector $s_{+}$is a multiple of the vector $g_{+}$, then (6) can also be written as $\alpha_{+}=\frac{s_{+}^{t} A s_{+}}{s_{+}^{t} s_{+}}$.

Despite the similarities between these two methods, Algorithm 2 is significantly faster than the steepest descent method at the same cost per iteration, see Barzilai and Borwein [2] and also Fletcher [4].

Barzilai and Borwein [2] presented a convergence analysis of their method only in the two-dimensional quadratic case. It is unlikely that their analysis can be extended to higher dimensional problems. In the present work, we establish the convergence of the Barzilai and Borwein gradient method for any strictly convex quadratic function.

This paper is organized as follows. In Section 2 we present a relationship between the gradient method for minimizing a quadratic function and the shifted power method. We believe that this connection is the key to understanding the convergence properties of the Barzilai and Borwein method. In Section 3 we study the convergence of their method applied to a quadratic function with a SPD Hessian. 


\section{Relationship to the Shifted Power Method}

Let us cousider the gradient method for problem (1) when $f$ is a differentiable function. For the purpose of comparison we will write the steplength choice in a slightly different way.

\section{Algorithm 3 (Gradient Method)}

Given $x_{0} \in I R^{n}$

For $k=0,1, \ldots$, (until convergence) $d o$

1. Choose steplength $\frac{1}{\alpha_{k}}$

2. Set $s_{k}=-\frac{1}{\alpha_{k}} \nabla f\left(x_{k}\right)$

3. Set $x_{k+1}=x_{k}+s_{k}$

End do

Both the steepest descent method and the Barzilai and Borwein method for quadratics are special cases of Algorithm 3. They differ only in the way the scalars $\alpha_{k}$ are chosen. Lemma 1 demonstrates a connection between Algorithm 3 and the shifted power method. This relationship will be used to establish our convergence results in Section 3.

Lemma 1 Let $f(x)=\frac{1}{2} x^{t} A x-b^{t} x+c$ where $A$ is a SPD matrix. Further let $x_{\star}$ be the unique minimizer of $f,\left\{x_{k}\right\}$ the sequence generated by Algorithm 3 and $\epsilon_{k}=x_{\star}-x_{k}$ for all $k$. Then

1. $A e_{k}=\alpha_{k} s_{k}$

2. $e_{k+1}=\frac{1}{\alpha_{k}}\left(\alpha_{k} I-A\right) e_{k}$

3. $s_{k+1}=\frac{1}{\alpha_{k+1}}\left(\alpha_{k} I-A\right) s_{k}$

Proof: Using the fact that $\nabla f\left(x_{k}\right)=A x_{k}-b$ and the definition of the step $s_{k}$ in Algorithm 3, the three claims in Lemma 1 follow directly 
Since $\mathrm{A}$ is SPD, the scalars $\alpha_{k}$ satisfy (5) when they are generated by either (6) or by (4). And so, claim 1 in Lemma 1 allows us to conclude that $\left\|c_{k}\right\|$ tends to zero if and only if $\left\|s_{k}\right\|$ tends to zero. Thus, for the minimizalion of a quadratic function with a SPD Hessian it suffices to study the behavior of $\left\{s_{k}\right\}$.

For any $s_{0}$, there exist constants $c_{1}, c_{2}, \ldots, c_{n}$ such that:

$$
s_{0}=\sum_{i=1}^{n} c_{i} v_{i}
$$

where $\left\{v_{1}, v_{2}, \ldots, v_{n}\right\}$ are orthonormal eigenvectors of $\mathrm{A}$ associated with the eigenvalues $\left\{\lambda_{1}, \lambda_{2}, \ldots, \lambda_{n}\right\}$. Now, using Lemma 1 it follows that for any integer $k$,

$$
s_{k+1}=\frac{1}{\gamma_{k}} \sum_{i=1}^{n}\left(\prod_{j=0}^{k}\left(\alpha_{j}-\lambda_{i}\right)\right) c_{i} v_{i}
$$

where

$$
\gamma_{k}=\prod_{j=1}^{k+1} \alpha_{j} .
$$

From (8) we can see that if we use the exact eigenvalues of $\mathrm{A}$ as the scalars $\alpha_{k}$ in Algorithm 3, in any order, then we find the exact solution in $p$ iterations, where $p$ is the number of distinct eigenvalues of A. Unfortunately, we do not know the eigenvalues of $\mathrm{A}$ in advance. However, we can choose $\alpha_{k}$ to be the Rayleigh quotient at $s_{k}$ (the choice of the steepest descent method) or the Rayleigh quotient at $s_{k-1}$ (the choice of the Barzilai and Borwein method) to approximate the eigenvalues of $A$. With the choice of the steepest descent method, it can be shown that $s_{k+1}^{t} s_{k}=0$ for all $k$, and also that the 2-norm of the error decreases at every iteration. Consequently, the scalars $\alpha_{k}$ tend to take values closer to $\lambda_{\text {max }}$ than to $\lambda_{\min }$. Therefore, the coefficients associated with the large eigenvalues in (8) will be effectively reduced, while the coefficients associated with the small eigenvalues only decrease very slowly. See Johnson [5] and Akaike [1] for details.

On the other hand, the choice of the Barzilai and Borwein method does not guarantee any orthogonality among the vectors $s_{k}$, and the 2-norm of the error might increase at some iterations. In fact, by properties of the Rayleigh quotient, the scalar $\alpha_{k}$ will approximate the eigenvalue associated with the largest coefficient in the eigenvector expansion of the vector $s_{k-1}$, 
regardless of the eigenvalue location in the spectrum. Therefore, the Barzilai and Borwein method is more effective at reducing the coefficients associated with all the eigenvalues in the eigenvector expansion (8).

The behavior of the sequence $\left\{\alpha_{k}\right\}$ for the Barzilai and Borwein method, in particular the approximation of the eigenvalues of the Hessian, is most easily appreciated by considering a particular example.

\section{Example 1}

Let $f(x)=\frac{1}{2} x^{t} A x$ where $A=\operatorname{diag}(1,2,12)$. Clearly, $f$ has a unique minimizer at $x_{\star}=(0,0,0)^{t}$. The first 10 iterations generated by Algorithm 2 starting at $x_{0}=(1,1,1)^{t}$ and $\alpha_{0}=1$ are shown in Table 1 . The Table lists the 2 -norm of the error, and the 2 -norm of the gradient. Also shown are the scalars $\alpha_{k}$ and the coefficients in the eigenvector expansion (8) associated with the three eigenvalues of $\mathrm{A}$.

Since $\alpha_{0}=\lambda_{1}=1$, the column with the coefficients of the eigenvector associated with $\lambda_{1}$ in Table 1 contains zero after the first iteration, and the scalar $\alpha_{k}$ approximates only the eigenvalues $\lambda_{2}=2$ and $\lambda_{3}=12 \mathrm{during}$ the rest of the process. Notice that $\alpha_{k}$ approximates, at each iteration, the eigenvalue with the larger coefficient in the previous iteration. In fact, the bigger the difference between the two coefficients the closer the scalar will be to the eigenvalue.

Notice also that this is not a descent algorithm. Under special circumstances, that will be studied in the next section, the 2-norm of the error $\epsilon_{k}$, as well as the objective function, increases at some iterations. This is in sharp contrast to the stcepest descent method. In fact, if the steepest descent method is used to minimize the function $f(x)$ with the same initial guess $x_{0}$, then 165 itcrations are required to achieve an error of $.3 \times 10^{-29}$. Observe that the Barzilai and Borwein method achieved this accuracy in only 10 iterations.

\section{Convergence Analysis}

In this section we establish the convergence of Algorithm 2 applied to any quadratic function with a SPD Hessian.

For any initial crror $e_{0}$, there exist constants $d_{1}^{0}, d_{2}^{0}, \ldots, d_{n}^{0}$ such that:

$$
c_{0}=\sum_{i=1}^{n} d_{i}^{0} v_{i}
$$




\begin{tabular}{|c|c|c|c|c|c|c|}
\hline \multirow{2}{*}{ iteration } & & \multirow{2}{*}{$\left\|\epsilon_{k}\right\|_{2}$} & $\left\|\nabla f\left(x_{k}\right)\right\|_{2}$ & $\alpha_{k}$ & \multicolumn{4}{|c|}{ coefficients of eigenvectors } \\
& & & & $\lambda_{1}=1$ & $\lambda_{2}=2$ & $\lambda_{3}=12$ \\
\hline 0 & $0.17 \mathrm{~d}+01$ & $0.12 \mathrm{~d}+02$ & $0.1000 \mathrm{~d}+01$ & $-.10 \mathrm{~d}+01$ & $-.20 \mathrm{~d}+01$ & $-.12 \mathrm{~d}+02$ \\
1 & $0.11 \mathrm{~d}+02$ & $0.13 \mathrm{~d}+03$ & $0.1165 \mathrm{~d}+02$ & $0.00 \mathrm{~d}+00$ & $0.17 \mathrm{~d}+00$ & $0.11 \mathrm{~d}+02$ \\
2 & $0.88 \mathrm{~d}+00$ & $0.42 \mathrm{~d}+01$ & $0.1199 \mathrm{~d}+02$ & $0.00 \mathrm{~d}+00$ & $0.14 \mathrm{~d}+00$ & $-.32 \mathrm{~d}+00$ \\
3 & $0.69 \mathrm{~d}+00$ & $0.13 \mathrm{~d}+01$ & $0.1045 \mathrm{~d}+02$ & $0.00 \mathrm{~d}+00$ & $0.13 \mathrm{~d}+00$ & $0.71 \mathrm{~d}-04$ \\
4 & $0.55 \mathrm{~d}+00$ & $0.11 \mathrm{~d}+01$ & $0.2000 \mathrm{~d}+01$ & $0.00 \mathrm{~d}+00$ & $0.56 \mathrm{~d}+00$ & $-.55 \mathrm{~d}-04$ \\
5 & $0.45 \mathrm{~d}-04$ & $0.54 \mathrm{~d}-03$ & $0.2000 \mathrm{~d}+01$ & $0.00 \mathrm{~d}+00$ & $0.80 \mathrm{~d}-06$ & $0.27 \mathrm{~d}-03$ \\
6 & $0.22 \mathrm{~d}-03$ & $0.27 \mathrm{~d}-02$ & $0.1199 \mathrm{~d}+02$ & $0.00 \mathrm{~d}+00$ & $0.65 \mathrm{~d}-14$ & $-.23 \mathrm{~d}-03$ \\
7 & $0.16 \mathrm{~d}-08$ & $0.19 \mathrm{~d}-07$ & $0.1200 \mathrm{~d}+02$ & $0.00 \mathrm{~d}+00$ & $0.54 \mathrm{~d}-14$ & $0.16 \mathrm{~d}-08$ \\
8 & $0.26 \mathrm{~d}-13$ & $0.53 \mathrm{~d}-13$ & $0.1200 \mathrm{~d}+02$ & $0.00 \mathrm{~d}+00$ & $0.45 \mathrm{~d}-14$ & $0.00 \mathrm{~d}+00$ \\
9 & $0.22 \mathrm{~d}-13$ & $0.44 \mathrm{~d}-13$ & $0.2000 \mathrm{~d}+01$ & $0.00 \mathrm{~d}+00$ & $0.22 \mathrm{~d}-13$ & $0.00 \mathrm{~d}+00$ \\
10 & $0.31 \mathrm{~d}-29$ & $0.63 \mathrm{~d}-29$ & $0.2000 \mathrm{~d}+01$ & $0.00 \mathrm{~d}+00$ & $-.32 \mathrm{~d}-29$ & $0.00 \mathrm{~d}+00$ \\
\hline
\end{tabular}

Table 1: Barzilai and Borwein method for Example 1

where $\left\{v_{1}, v_{2}, \ldots, v_{n}\right\}$ are orthonormal eigenvectors of $A$ associated with the eigenvalues $\left\{\lambda_{1}, \lambda_{2}, \ldots, \lambda_{n}\right\}$.

Using Lemma 1 we obtain for any integer $k$,

$$
\epsilon_{k+1}=\sum_{i=1}^{n} d_{i}^{k+1} v_{i}
$$

where

$$
d_{i}^{k+1}=\prod_{j=0}^{k}\left(\frac{\alpha_{j}-\lambda_{i}}{\alpha_{j}}\right) d_{i}^{0} .
$$

We observe that the convergence properties of the sequence $\left\{c_{k}\right\}$ will depend on the behavior of each one of the sequences $\left\{d_{i}^{k}\right\}, 1 \leq i \leq n$. Later in this section, we will prove that each of these sequences converges to zero. First let us establish the Q-linear convergence of Algorithm 2 applied to a quadratic function with a SPD Hessian that satisfies the admittedly restrictive condition

$$
\lambda_{\max }<2 * \lambda_{\min }
$$


Lemma 2 Let $f(x)=\frac{1}{2} x^{t} A x-b^{t} x+c$ where $A$ is SPD and satisfies (10). Let $\left\{x_{k}\right\}$ be the scquence gencraled by Algorithm 2 and $x_{\star}$ the unique minimizer of $f$. Then the sequence $\left\{x_{k}\right\}$ converges $Q$-linearly to $x_{\star}$ in the Euclidean norm with convcrgence factor $\hat{c}=\left(\lambda_{\max }-\lambda_{\min }\right) / \lambda_{\min }$.

Proof: Using Lemma 1 and (9) we obtain for any $k$,

$$
e_{k+1}=\frac{1}{\alpha_{k}}\left(\alpha_{k} I-A\right) \sum_{i=1}^{n} d_{i}^{k} v_{i}=\sum_{i=1}^{n}\left(\frac{\alpha_{k}-\lambda_{i}}{\alpha_{k}}\right) d_{i}^{k} v_{i}
$$

By the orthonormality of the eigenvectors we have

$$
\left\|\epsilon_{k+1}\right\|_{2}^{2}=\sum_{i=1}^{n}\left(d_{i}^{k}\right)^{2}\left(\frac{\alpha_{k}-\lambda_{i}}{\alpha_{k}}\right)^{2} \leq \max _{i}\left(\frac{\alpha_{k}-\lambda_{i}}{\alpha_{k}}\right)^{2}\left\|c_{k}\right\|_{2}^{2} .
$$

From (10), recalling that $\alpha_{k}$ obeys (5),

$$
\max _{i}\left|\frac{\alpha_{k}-\lambda_{i}}{\alpha_{k}}\right| \leq \frac{\lambda_{\max }-\lambda_{\min }}{\lambda_{\min }}<1 .
$$

Combining (11) and (12) gives

$$
\left\|e_{k+1}\right\|_{2} \leq \hat{c}\left\|e_{k}\right\|_{2} \quad \text { where } \quad \hat{c}=\frac{\lambda_{\max }-\lambda_{\min }}{\lambda_{\min }}<1
$$

Now we can explain why the norm of the error might increase at some iterations when the spectrum of A does not satisfy (10). Let us first divide the spectrum of $A$ into two subintervals

$$
\text { Left }=\left[\lambda_{\min }, \frac{\lambda_{\max }}{2}\right] \text { and Right }=\left(\frac{\lambda_{\max }}{2}, \lambda_{\max }\right] .
$$

Clearly, if the spectrum of A obeys (10) then the Left interval is empty and Lemma 2 says that the error decreases at each iteration. If we force the scalars $\alpha_{k}$ to be in the Right interval, by a similar argument, $\left\{x_{k}\right\}$ converges Q-linearly to $x_{\star}$ for any $x_{0} \in I R^{n}$. But Algorithm 2 moves the scalars $\alpha_{k}$ dynamically within the spectrum of $A$. If at the $j^{\text {th }}$ iteration $\alpha_{j} \in$ Left , then the coefficient associated with the eigenvalues $\lambda_{i}$ that satisfy $\lambda_{i}>2 \alpha_{j}$, will be amplified by the factor $\left|\frac{\alpha_{j}-\lambda_{i}}{\alpha_{j}}\right|>1$ (i.e., $\left|d_{i}^{j+1}\right|>\left|d_{i}^{j}\right|$ ), and this could lead to an increase of $\left\|\epsilon_{j+1}\right\|$ with respect to $\left\|\epsilon_{j}\right\|$. In general, the sequences $\left\{d_{i}^{k}\right\}$ defined in (9) will increase at some iterations. However, the sequence $\left\{d_{1}^{k}\right\}$ associated with the eigenvalue $\lambda_{\min }$ will decrease at every itcration. 
Lemma 3 The sequence $\left\{d_{1}^{k}\right\}$ converges to zero Q-linearly with convergence factor $\hat{c}=1-\left(\lambda_{\min } / \lambda_{\max }\right)$.

Proof: For any positive integer $k$,

$$
d_{1}^{k+1}=\left(\frac{\alpha_{k}-\lambda_{\min }}{\alpha_{k}}\right) d_{1}^{k}
$$

Since $\alpha_{k}$ satisfies (5), we have

$$
0<\frac{\lambda_{\min }}{\lambda_{\max }} \leq \frac{\lambda_{\min }}{\alpha_{k}} \leq 1
$$

And so,

$$
\left|d_{1}^{k+1}\right|=\left(1-\frac{\lambda_{\min }}{\alpha_{k}}\right)\left|d_{1}^{k}\right| \leq \hat{c}\left|d_{1}^{k}\right|
$$

where

$$
\hat{c}=1-\frac{\lambda_{\min }}{\lambda_{\max }}<1
$$

In the proof of our convergence theorem, we will use the following result.

Lemma 4 If the sequences $\left\{d_{1}^{k}\right\},\left\{d_{2}^{k}\right\}, \ldots,\left\{d_{l}^{k}\right\}$ all converge to zcro for a fixed integer $l, 1 \leq l<n$. Then,

$$
\liminf _{k \rightarrow \infty}\left|d_{l+1}^{k}\right|=0
$$

Proof: Suppose, by way of contradiction, that there exists a constant $\epsilon>0$ such that

$$
\left(d_{l+1}^{k}\right)^{2} \lambda_{l+1}^{2}>\epsilon \text { for all } k .
$$

By (9), Lemma 1 and the orthonormality of the eigenvectors $\left\{v_{1}, v_{2}, \ldots, v_{n}\right\}$, we can see that the Rayleigh quotient $\alpha_{k+1}$ can be written as

$$
\alpha_{k+1}=\frac{\sum_{i=1}^{n}\left(d_{i}^{k}\right)^{2} \lambda_{i}^{3}}{\sum_{i=1}^{n}\left(d_{i}^{k}\right)^{2} \lambda_{i}^{2}} .
$$

Since the sequences $\left\{d_{1}^{k}\right\}, \ldots,\left\{d_{l}^{k}\right\}$ all converge to zero, there exists $\hat{k}$ sufficiently large such that

$$
\sum_{i=1}^{l}\left(d_{i}^{k}\right)^{2} \lambda_{i}^{2}<\frac{\epsilon}{2} \text { for all } k \geq \hat{k}
$$


By (13) and (14), we obtain

$$
\frac{\left(\sum_{i=l+1}^{n}\left(d_{i}^{k}\right)^{2} \lambda_{i}^{2}\right) \lambda_{l+1}}{\frac{\epsilon}{2}+\left(\sum_{i=l+1}^{n}\left(d_{i}^{k}\right)^{2} \lambda_{i}^{2}\right)} \leq \alpha_{k+1} \leq \lambda_{\max } .
$$

Since

$$
\sum_{i=l+1}^{n}\left(d_{i}^{k}\right)^{2} \lambda_{i}^{2} \geq\left(d_{l+1}^{k}\right)^{2} \lambda_{l+1}^{2}>\epsilon
$$

then, by using (15), it follows that

$$
\frac{2}{3} \lambda_{l+1} \leq \alpha_{k+1} \leq \lambda_{\max } \text { for all } k \geq \hat{k}
$$

Finally, using the fact that $d_{l+1}^{k+1}=\left(\left(\alpha_{k}-\lambda_{l+1}\right) / \alpha_{k}\right) d_{l+1}^{k}$, we obtain for all $k \geq \hat{k}+1$,

$$
\left|d_{l+1}^{k+1}\right|=\left|1-\frac{\lambda_{l+1}}{\alpha_{k}}\right|\left|d_{l+1}^{k}\right| \leq \hat{c}\left|d_{l+1}^{k}\right|
$$

where

$$
\hat{c}=\max \left(\frac{1}{2}, 1-\frac{\lambda_{l+1}}{\lambda_{\max }}\right)<1,
$$

which is a contradiction. Therefore, $\liminf _{k \rightarrow \infty}\left|d_{l+1}^{k}\right|=0$

Theorem 1 establishes the convergence of the Barzilai and Borwein method when applied to a quadratic function with a SPD Hessian.

Theorem 1 Lct $f(x)$ be a strictly convex quadratic function. Let $\left\{x_{k}\right\}$ be the sequence generaled by Algorithm $\stackrel{2}{2}$ and $x_{\star}$ the unique minimizer of $f$. Then, either $x_{j}=x_{\star}$ for some finite $j$, or the sequence $\left\{x_{k}\right\}$ converges to $x_{\star}$.

Proof: We need only consider the case in which there is no finite integer $j$ such that $x_{j}=x_{\star}$. Hence, it suffices to prove that the sequence $\left\{\epsilon_{k}\right\}$ converges to zero.

From (9) and the orthonormality of the eigenvectors we have

$$
\left\|c_{k}\right\|_{2}^{2}=\sum_{i=1}^{n}\left(d_{i}^{k}\right)^{2}
$$


And so, the sequence of errors $\left\{e_{k}\right\}$ converges to zero if and only if each one of the sequences $\left\{d_{i}^{k}\right\}$ for $i=1,2, \ldots, n$ converges to zero.

Suppose, by way of contradiction, that some of the sequences $\left\{d_{i}^{k}\right\}$ are not converging to zero. In particular, let us suppose that $p$ is the smallest integer between 1 and $n$ for which the sequence $\left\{d_{p}^{k}\right\}$ does not converge to zero. By Lemma 3, we can see that $p \geq 2$.

Since $\left\{d_{1}^{k}\right\}, \ldots\left\{d_{p-1}^{k}\right\}$ all converge to zero, then for a given $\epsilon>0$ there exists $\hat{k}$ sufficiently large such thai.

$$
\sum_{i=1}^{p-1}\left(d_{i}^{k}\right)^{2} \lambda_{i}^{2}<\frac{\epsilon}{2} \text { for all } k \geq \hat{k} .
$$

By Lemma 4, it follows that $\liminf \operatorname{in}_{k \rightarrow \infty}\left|d_{p}^{k}\right|=0$. Hence, there exists $k_{p} \geq \hat{k}$ such that.

$$
\left(d_{p}^{k_{p}}\right)^{2} \lambda_{p}^{2}<\epsilon .
$$

Now, to study the behavior of the sequence $\left\{d_{p}^{k}\right\}$ for $k \geq k_{p}$, we define

$$
M_{\epsilon}=\sup _{i \geq k_{p}}\left\{\left(d_{p}^{i}\right)^{2}\right\}
$$

Let us say that $k_{0}>k_{p}$ is any positive integer for which $\left(d_{p}^{k_{0}-1}\right)^{2} \lambda_{p}^{2}<\epsilon$ and $\left(d_{p}^{k_{0}}\right)^{2} \lambda_{p}^{2}>\epsilon$. From (13) and (16), it follows that

$$
\frac{2}{3} \lambda_{p} \leq \alpha_{k} \leq \lambda_{\max }
$$

for all integer $k$ that satisfies $k_{0}+1 \leq k \leq j$, where $j$ is the first positive integer greater than $k_{0}$ for which $\left(d_{p}^{j}\right)^{2} \lambda_{p}^{2}<\epsilon$.

Finally, using the bound

$$
\left|d_{p}^{k_{0}+1}\right| \leq\left(\frac{\lambda_{\max }-\lambda_{\min }}{\lambda_{\min }}\right)^{2}\left|d_{p}^{k_{0}-1}\right|
$$

and the fact that

$$
\left|d_{p}^{k+1}\right| \leq \max \left(\frac{1}{2}, 1-\frac{\lambda_{p}}{\lambda_{\max }}\right)\left|d_{p}^{k}\right|,
$$

whenever $\alpha_{k}$ satisfies (17), we conclude that

$$
M_{\epsilon} \leq\left(\frac{\lambda_{\max }-\lambda_{n i n}}{\lambda_{\min }}\right)^{4} \frac{\epsilon}{\lambda_{p}^{2}} .
$$


Since $\epsilon>0$ can be chosen arbitrary small, then $\limsup _{k \rightarrow \infty}\left(d_{p}^{k}\right)^{2}=0$. Hence, $\lim _{k \rightarrow \infty}\left|d_{p}^{k}\right|=0$, which is a contradiction. Therefore, the sequence $\left\{c_{k}\right\}$ converges to zero

Notice that with the choice of $\hat{\alpha}_{k+1}=\frac{s_{k}^{t} A^{2} s_{k}}{s_{k}^{t} A s_{k}}$ instead of $\alpha_{k+1}=\frac{s_{k}^{t} A s_{k}}{s_{k}^{t} s_{k}}$, equality (13) can be written as

$$
\hat{\alpha}_{k+1}=\frac{\sum_{i=1}^{n}\left(d_{i}^{k}\right)^{2} \lambda_{i}^{4}}{\sum_{i=1}^{n}\left(d_{i}^{k}\right)^{2} \lambda_{i}^{3}} .
$$

Then, by a similar argument, we conclude that the convergence result established in Theorem 1 for Algorithm 2 with the choice of $\alpha_{k+1}$ also holds with the choice of $\hat{\alpha}_{k+1}$.

\section{Acknowledgements}

The author wishes to thank Richard Byrd, John E. Dennis and Richard A. Tapia for helpful comments and suggestions. 


\section{References}

[1] H. AKAIKE. On a successive transformation of probability distribution and its application to the analysis of the optimum gradient method. Ann. Inst. Statist. Math. Tokyo, 11:1-17, 1959.

[2] J. BBARZILAI and J.M. BORWEIN. Two point step size gradient methods. IMA Journal of Numerical Analysis, 8:141-148, 1988.

[3] J.E. DENNIS Jr. and R.B. SCHNABEL. Numerical Methods for Unconstrained Optimization and Nonlinear Equations. Prentice-Hall, Englewood Cliffs, NJ, 1983.

[1] R. FLETCHER. Low storage methods for unconstrained optimization. Lectures in Applicd Mathematics (AMS), 26:165-179, 1990.

[5] CLAES JOHNSON. Numerical Solution of Partial Differential Equations by the Finite Element Method. Cambridge University Press, 1990. 